\title{
Linear chemically sensitive electron tomography using DualEELS and compressed sensing
}

\author{
Alaa AlAfeef ${ }^{1,2}$, W. Paul Cockshott ${ }^{1}$, Patrick Barges ${ }^{2}$, Ian Zuazo ${ }^{2}$, Joanna Bobynko ${ }^{3}$, Alan J. Craven ${ }^{3}$, \\ and Ian Maclaren ${ }^{3}$ \\ ${ }^{1}$ School of Computing Science, University of Glasgow, Glasgow, G12 8QQ, UK \\ 2 ArcelorMittal Maizières Research, Maizières-lès-Metz, 57283, France \\ ${ }^{3}$ SUPA School of Physics and Astronomy, University of Glasgow, Glasgow, G12 8QQ, UK
}

Electron tomography (ET) is now increasingly important for recovering the three-dimensional (3D) morphology of nanostructured materials in the physical and life sciences. ET typically involves the acquisition of a set of two-dimensional projection images at different tilts using (scanning) transmission electron microscopy ([S]TEM), followed by alignment and reconstruction using established algorithms to reconstruct a 3D volume that represent the physical morphology or 3D distribution of some other property of the specimen under investigation. In principle, the methodology is independent of the nature of the images and is applicable to any imaging technique that fulfils the projection requirement [1] such that the signal should change monotonically with the physical property of the sample. This condition is approximately fulfilled for mass-thickness contrast in bright field TEM of amorphous biological specimens, and high angle annular dark field (HAADF) STEM imaging of thin specimens. Consequently, both imaging techniques have been widely used in ET.

Recently, ET has been performed using spectroscopic signals, including X-ray spectroscopy, energyfiltered TEM (EFTEM), and electron energy loss spectroscopy (EELS) in the STEM, to achieve a chemically sensitive 3D reconstruction. Whilst X-rays and EFTEM mainly allow the mapping of elemental contents, EELS offers additional possibilities for studying detailed chemistry. Early studies have already shown the feasibility of EELS-STEM tomography [2,3]. Nevertheless, EELS suffers significantly from multiple scattering, especially for thicker specimens, and this makes the backgroundsubtracted edge signal a non-linear function of thickness, which leads to reconstruction artifacts.

Using DualEELS we can simultaneously record the low- and high-loss EELS signals for each pixel in a spectrum image, and this allows the subsequent deconvolution of the multiple scattering out of the highloss signal. This gives edges with integrated intensities that have a clear linear relationship to thickness. This was performed on $\mathrm{VC}_{\mathrm{x}}$ particles on a carbon extraction replica of a vanadium microalloyed highmanganese steel. DualEELS spectrum images (SIs) were recorded at approximately $10^{\circ}$ intervals from about $-50^{\circ}$ to $+50^{\circ}$ using a JEOL ARM200F equipped with a Gatan GIF Quantum ER spectrometer. The data was processed by energy alignment of all SIs using the zero loss peak, noise reduction using principal component analysis, deconvolution to remove plural scattering, and production of vanadium maps with a $50 \mathrm{eV}$ integration window using a recently developed procedure [4]. 3D reconstruction was performed using both the well-known SIRT algorithm, as well a new compressed-sensing based algorithm, DLET [5], which is a dictionary-based and very noise tolerant algorithm that can obtain highfidelity reconstructions from far fewer projections than are normally required by traditional ET methods.

Figure 1 shows a comparison between SIRT and DLET for reconstruction using the V-L $\mathrm{L}_{2,3}$ EELS signal. It can be noted that DLET gives a noticeable reduction in $\mathbf{z}$-elongation due to missing wedge artifacts, which is a common feature of tomographic reconstructions (although this elongation is not totally 
eliminated), as well as reduced noise within and outwith the precipitate. Figure 2 shows line profiles through two orthoslices for both this EELS reconstruction of Figure 1, as well as a 3D reconstruction from the HAADF signal recorded simultaneously with the EELS spectrum image. These both show a very flat profile through the centre of the particle, and no apparent hollowing ( "cupping" artifact), which would be a signature of a non-linear relationship between signal and thickness. Thus, appropriate use of DualEELS enables linear chemically sensitive tomography on nanoparticles of an industrially important material. This combined with a new compressed sensing based algorithm, DLET, allows high-fidelity, low-noise reconstruction from relatively few projections and minimizes artifacts from the missing wedge in the tilt data. This could easily be extended to encompass changes in near-edge EELS fine structure in order to produce 3D views of chemical form or bonding in nanostructured materials.

\section{References:}

[1] P. W Hawkes, in "Electron tomography, $2^{\text {nd }}$ edition", ed. J. Frank (Springer, New York).

[2] K. Jarausch et al., Ultramicroscopy 109 (2009) p. 326.

[3] L.Yedra, et al., Ultramicroscopy 122 (2012) p.12-18.

[4] J. Bobynko, I. MacLaren, and A. J. Craven, Ultramicroscopy 149 (2015) p. 9.

[5] A. AlAfeef et al., J. Phys.: Conf. Ser 522 (2014) P. 012021.

[6] AAA acknowledges funding for a PhD studentship from the Lord Kelvin/Adam Smith Scholarship of the University of Glasgow. The work on high-Mn steels was funded by the EU Research Fund for Coal and Steel (Precipitation in High Manganese Steels, RFSR-CT-2010-00018). This work was made possible by generous provision of the MagTEM facility by SUPA and the University of Glasgow.
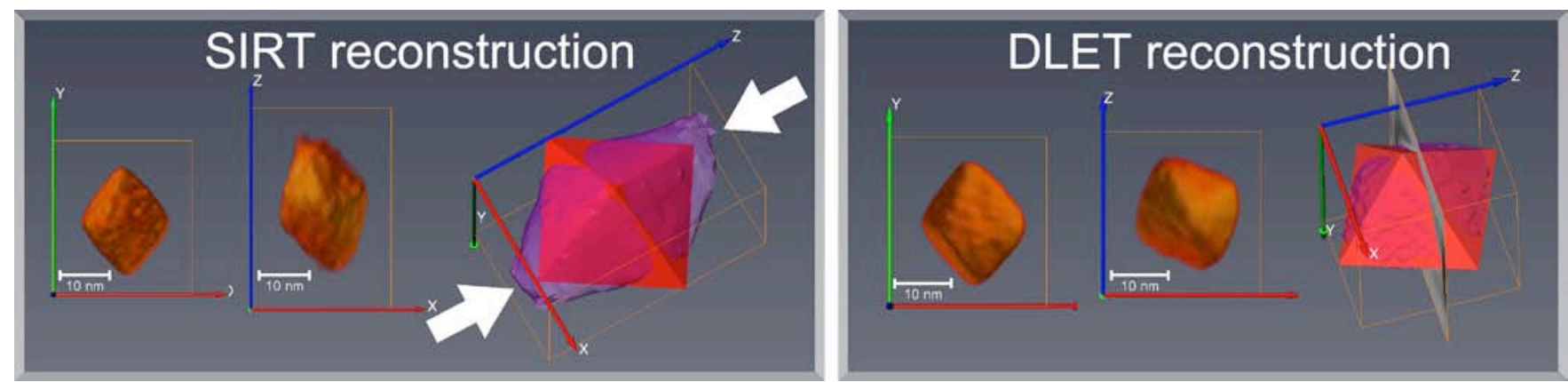

Figure 1. A comparison of reconstructions of one $\mathrm{VC}_{\mathrm{x}}$ precipitate from the $\mathrm{V}-\mathrm{L}_{2,3}$ signal using SIRT and the new DLET algorithm showing X-Y, and X-Z views, as well as a comparison to an idealized octahedron. Note that the distortion in the $\mathrm{Z}$ (missing wedge) direction is much reduced using DLET.
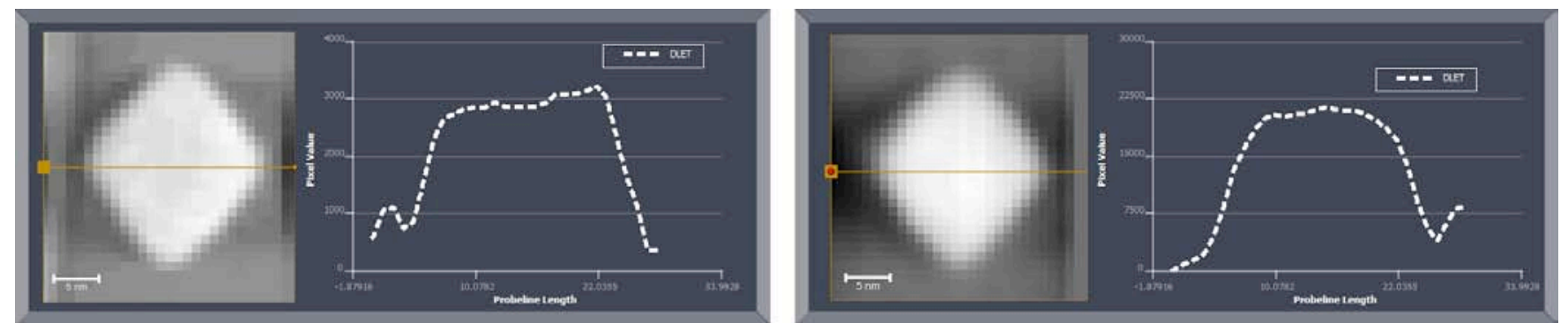

Figure 2. Orthoslices through the centre of two reconstructions of a precipitate made using (Left) the V-L ${ }_{2,3}$ EELS signal, and (Right) the HAADF signal recorded simultaneously. Both were reconstructed using the DLET algorithm. In both cases, line profiles of density are shown corresponding to a horizontal line through the center of Orthoslices and neither show any cupping artifacts. 